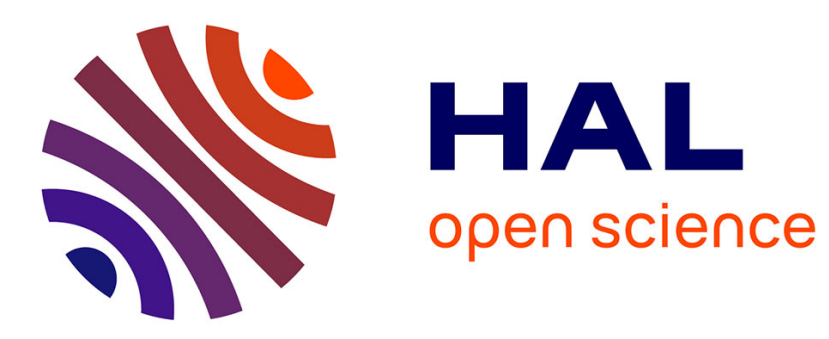

\title{
Possibility of long-distance heat transport in weightlessness using supercritical fluids
}

Daniel Beysens, Denis Chatain, Vadim Nikolayev, Jalil Ouazzani, Yves

Garrabos

\section{- To cite this version:}

Daniel Beysens, Denis Chatain, Vadim Nikolayev, Jalil Ouazzani, Yves Garrabos. Possibility of longdistance heat transport in weightlessness using supercritical fluids. Physical Review E: Statistical, Nonlinear, and Soft Matter Physics, 2010, 82 (6), 061126 (11 p.). 10.1103/PhysRevE.82.061126. hal-00551878

\section{HAL Id: hal-00551878 \\ https://hal.science/hal-00551878}

Submitted on 1 Jul 2021

HAL is a multi-disciplinary open access archive for the deposit and dissemination of scientific research documents, whether they are published or not. The documents may come from teaching and research institutions in France or abroad, or from public or private research centers.
L'archive ouverte pluridisciplinaire HAL, est destinée au dépôt et à la diffusion de documents scientifiques de niveau recherche, publiés ou non, émanant des établissements d'enseignement et de recherche français ou étrangers, des laboratoires publics ou privés. 


\title{
Possibility of long-distance heat transport in weightlessness using supercritical fluids
}

\author{
D. Beysens, ${ }^{1,2}$ D. Chatain, ${ }^{1}$ V. S. Nikolayev, ${ }^{1,2}$ J. Ouazzani, ${ }^{3}$ and Y. Garrabos ${ }^{4}$ \\ ${ }^{1}$ Service des Basses Températures, INAC/CEA, 17 rue des Martyrs, 38054 Grenoble Cedex 9, France \\ ${ }^{2}$ ESEME, PMMH-ESPCI, 10 rue Vauquelin, 75231 Paris Cedex 5, France \\ ${ }^{3}$ SARL ArcoFluid, Parc Unitec 1, 4 Allée du Doyen Georges Brus, 33600 Pessac, France \\ ${ }^{4}$ Institut de Chimie de la Matière Condensée de Bordeaux, UPR 9048, CNRS, 87 Avenue du Dr. Schweitzer, 33608 Pessac Cedex, France
}

(Received 23 July 2010; published 21 December 2010)

\begin{abstract}
Heat transport over large distances is classically performed with gravity or capillarity driven heat pipes. We investigate here whether the "piston effect," a thermalization process that is very efficient in weightlessness in compressible fluids, could also be used to perform long-distance heat transfer. Experiments are performed in a modeling heat pipe (16.5 mm long, $3 \mathrm{~mm}$ inner diameter closed cylinder), with nearly adiabatic polymethylmethacrylate walls and two copper base plates. The cell is filled with $\mathrm{H}_{2}$ near its gas-liquid critical point (critical temperature: $33 \mathrm{~K}$ ). Weightlessness is achieved by submitting the fluid to a magnetic force that compensates gravity. Initially the fluid is isothermal. Then heat is sent to one of the bases with an electrical resistance. The instantaneous amount of heat transported by the fluid is measured at the other end. The data are analyzed and compared with a two-dimensional numerical simulation that allows an extrapolation to be made to other fluids (e.g., $\mathrm{CO}_{2}$, with critical temperature of $300 \mathrm{~K}$ ). The major result is concerned with the existence of a very fast response at early times that is only limited by the thermal properties of the cell materials. The yield in terms of ratio, injected or transported heat power, does not exceed 10-30\% and is limited by the heat capacity of the pipe. These results are valid in a large temperature domain around the critical temperature.
\end{abstract}

DOI: 10.1103/PhysRevE.82.061126

PACS number(s): 05.60.-k, 05.70.Jk, 44.35.+c, 68.03.Cd

\section{INTRODUCTION}

Heat transport over large distances is classically performed by the latent heat transport. Conduction is effective only on short distances because of the slowness of the heat diffusion process. In the 1990s, a new way of the heat transfer in very compressible fluids has been evidenced. This phenomenon, which is particularly important under weightlessness, has been called piston effect [1-3]. When heating a fluid confined in a container, a thin hot boundary layer (HBL) of thickness $\delta_{1}$ forms close to the heating wall [4]. This layer expands and at the same time compresses adiabatically the rest of the fluid. As a result, a spatially uniform heating of the bulk fluid is achieved in a very short time.

The heat flux is limited by the temperature gradient in the heating wall and in the fluid HBL. If the other side of the sample, located at a distance $L$ from the heating wall, is colder, a cold boundary layer (CBL) of length $\delta_{2}$ will simultaneously form at the corresponding wall [see Fig. 1(c) below]. In between, the temperature is homogeneous. A heat transport can thus be performed in both HBL and CBL, connected by the bulk fluid that acts as a kind of thermal short circuit. While such a "heat pipe" does not work in the stationary regime (the piston effect concerns only transient behavior), it could serve to quickly transfer heat.

Studies of transient heat flux in the presence of piston effect have already been performed in ${ }^{3} \mathrm{He}$ under Earth's gravity in a flat sample of large aspect ratio (thickness of 1 $\mathrm{mm}$ and diameter of $57 \mathrm{~mm}$ ) perpendicular to gravity for Rayleigh numbers below and above the convection threshold [5] and in $\mathrm{CO}_{2}$ under weightlessness [6]. Here, in order to evaluate whether this process can be used to transport heat on large distances under weightlessness, experiments are performed under the conditions of magnetic gravity compensa- tion in a cylindrical cell with the largest length that preserves magnetic compensation (aspect ratio of 0.182). The cell is filled with $\mathrm{H}_{2}$ near its gas-liquid critical point (critical temperature $\left.T_{c}=33 \mathrm{~K}\right)$.

\section{EXPERIMENTAL SETUP}

Weightlessness conditions are achieved by using magnetic forces to compensate the gravity. The magnetic gravity compensation technique is recently reviewed in [7]. The HYLDE installation (hydrogen levitation device) situated at CEA/ Grenoble has been used here. It is described in detail in $[8,9]$.

\section{A. Cell}

The fluid is confined in a cylinder of $L=16.5 \mathrm{~mm}$ long, 3 $\mathrm{mm}$ inner diameter, and $4 \mathrm{~mm}$ outer diameter made of polymethylmetacrylate $(\mathrm{PMMA}=$ Plexiglas, denoted below with the subscript $P A$ ) (Fig. 1). The cylinder axis coincides with the magnetic coil axis. PMMA has been chosen because it is transparent and has a low thermal conductivity $\left(k_{P A}\right.$ $=0.125 \mathrm{~W} \mathrm{~m}^{-1} \mathrm{~K}^{-1}$ at $33 \mathrm{~K}$ ) and the specific heat of the walls is low $\left(C_{P A}=180 \mathrm{~J} \mathrm{~kg}^{-1} \mathrm{~K}^{-1}\right.$ at $\left.33 \mathrm{~K}\right)$. With a mass density of $1.15 \times 10^{3} \mathrm{~kg} \mathrm{~m}^{-3}$, the thermal diffusivity is $D_{P A}=6.04 \times 10^{-7} \mathrm{~m}^{2} \mathrm{~s}^{-1}$, corresponding to the characteristic diffusion time $t_{P A}=L^{2} / D_{P A}=450 \mathrm{~s}$. The PMMA cylinder is sealed with stainless-steel rings to two parallel electrolytic copper (thermal conductivity at $33 \mathrm{~K}$ is $1130 \mathrm{~W} \mathrm{~m}^{-1} \mathrm{~K}^{-1}$ ) blocks. They are in thermal contact with the helium bath by thermal conductors. The temperatures of the blocks are measured with Cernox ${ }^{\circledR}$ thermistors. They are made of a composite that provides a very weak resistance variation with the magnetic field. Their time constant is on the order of $20 \mathrm{~ms}$. Stainless steel is a thermal insulator at these low tempera- 
(a)

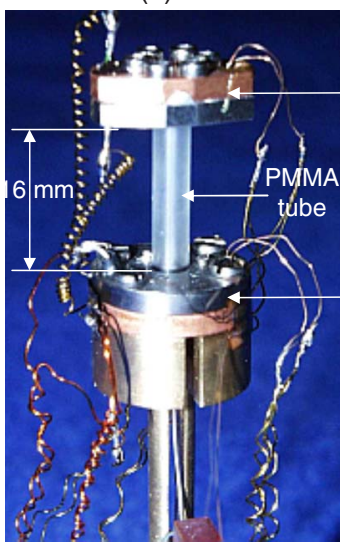

(b)

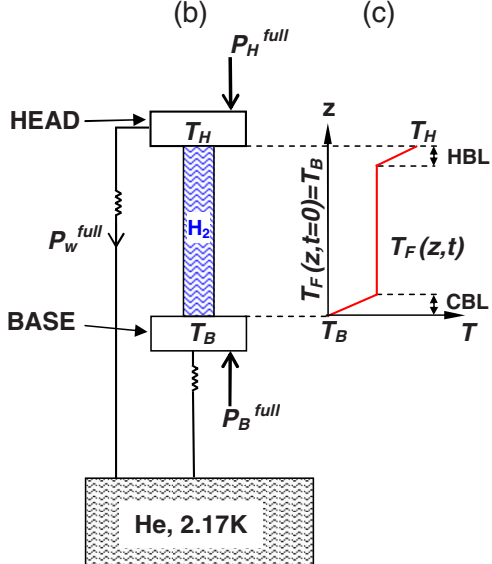

FIG. 1. (Color online) The experimental piston effect heat pipe. (a) photo; (b) heat exchange model. Both the head and the base are connected to the helium bath via heat conductors (thin wires). The heat power $P_{w}^{\text {full }}$ leaks from the head to the bath (see Sec. III A 2). The controlled heat power is supplied to the head $\left(P_{H}^{f u l l}\right)$ and to the base $\left(P_{B}^{\text {full }}\right)$. (c) Schematic instantaneous fluid temperature variation provided by the piston effect. The cold (CBL) and hot (HBL) boundary layers are indicated.

tures (thermal conductivity is $3.37 \mathrm{~W} \mathrm{~m}^{-1} \mathrm{~K}^{-1}$ ). The mass of the upper copper block (hot part), called the "head," is $m$ $=2.37 \mathrm{~g}$. The other block, the "base" (the coldest part), is kept at constant temperature $T_{B}$ by a closed-loop temperature regulation, the proportional integral differential (PID) device. The temperature control accuracy is $\pm 1 \mathrm{mK}$ at $33 \mathrm{~K}$ and the working temperature range is $15-40 \mathrm{~K}$.

The cell can be filled in situ with pure pressurized $\mathrm{H}_{2}$ through a capillary. This capillary is closed by a $\mathrm{H}_{2}$ ice plug $\left(\mathrm{H}_{2}\right.$ solidification temperature is $\left.14 \mathrm{~K}\right)$, whose formation is provided locally by a thermal conductor in contact with the helium bath. The cell is observed by light transmission. Parallel light is directed with mirrors from outside the anticryostat to the sample. The beam is parallel to the axis of the cylindrical cell and is deflected by mirrors to the camera outside the anticryostat. The whole setup is situated in a vacuum chamber.

At room temperature and at equilibrium, $\mathrm{H}_{2}$ is actually a mixture of three volumes of orthohydrogen (parallel spins of $\mathrm{H}$ nuclei, $o-\mathrm{H}_{2}$ ) with one volume of parahydrogen (antiparallel spins, $p-\mathrm{H}_{2}$ ). This gas is called normal hydrogen, $n-\mathrm{H}_{2}$. When $\mathrm{H}_{2}$ is cooled, the $o-\mathrm{H}_{2}-p-\mathrm{H}_{2}$ equilibrium is shifted and almost all $o-\mathrm{H}_{2}$ gradually transforms to $p-\mathrm{H}_{2}$ (more precisely, $96 \%$ of $p-\mathrm{H}_{2}$ at equilibrium at $30 \mathrm{~K}$ ). The exact critical pressure, temperature, and density of $p-\mathrm{H}_{2}$ are $T_{c}$ $=32.976 \mathrm{~K}, p_{c}=1.2928 \mathrm{MPa}$, and $\rho_{c}=31.426 \mathrm{~kg} \mathrm{~m}^{-3}$ [8]. The cell is filled at critical density $\rho_{c}$ by checking that the gas-liquid meniscus does not displace in the cell while approaching $T_{c}$ from below. The precision is on the order of $\pm 0.5 \mathrm{~mm}$ which corresponds to the $\pm 3 \%$ uncertainty of the critical density. The precision of the determination of $T_{c}$ is within $1 \mathrm{mK}$. This value shifts with the conversion $o-\mathrm{H}_{2}-p-\mathrm{H}_{2}$ with a time constant of order $50 \mathrm{~h}$ [8]. Then the experiments close to $T_{c}$ are performed more than 2 days after the beginning of the runs with the value $T_{c}$ checked before and after these runs.

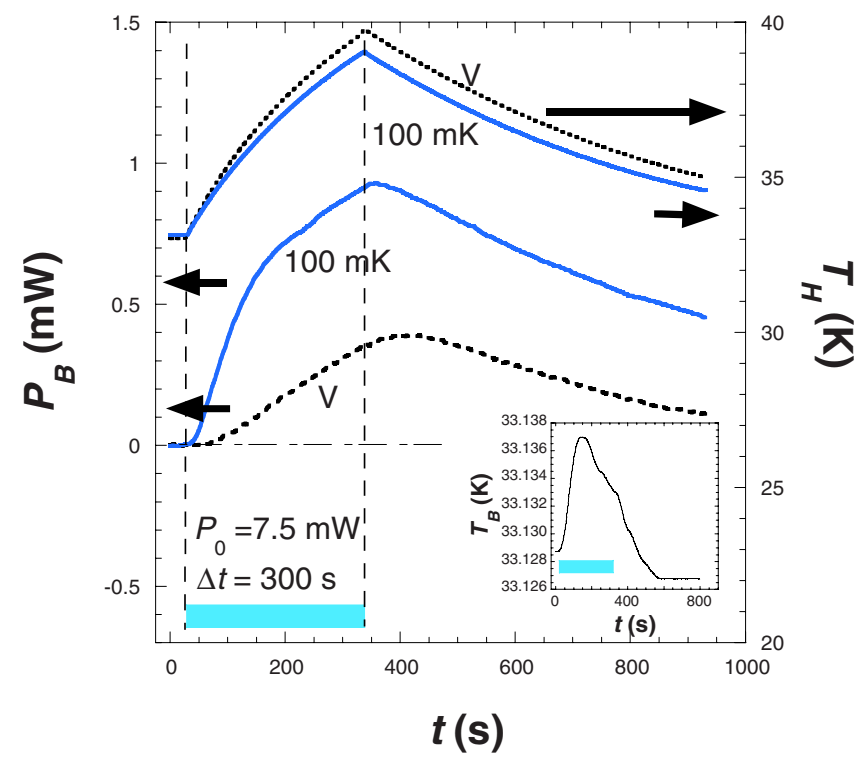

FIG. 2. (Color online) Typical measurements of the transmitted heat flux $\left(P_{B}\right)$, head temperature $\left(T_{H}\right)$, and base temperature $\left(T_{B}\right.$, in the inset) when a $7.5 \mathrm{~mW}$ power is injected into the head during about $300 \mathrm{~s}$ (shown with a bar). Solid lines: filled cell, $T_{0}=T_{c}$ +100 mK; dotted lines: empty cell (under vacuum).

\section{B. Measurement procedure}

Initially, the base, the head, the fluid, and the cell walls are at the same temperature $T_{0}$. The head temperature is maintained constant by sending a constant power $(\sim 5 \mathrm{~mW})$ that compensates the heat losses through the wiring (Fig. 1). It is important to precisely calibrate the head and the base temperature sensors. We took benefit of the strong convective flows that form a few millikelvins to $T_{c}$ under gravity in the presence of the slightest temperature gradients. Head $\left(T_{H}\right)$ and base $\left(T_{B}\right)$ temperatures are assumed to be equal when, under Earth's gravity, the convection disappears. Then the magnetic field is switched on and further measurements are performed at weightlessness.

The electric power $P_{B}^{f u l l}$ is supplied to the cell base by the temperature regulation that maintains $T_{B}$ constant. $P_{B}^{\text {full }}$ $(t=0) \approx 200 \mathrm{~mW}$ when the head and the base are at the same temperature. The power $P_{H}^{f u l l}$ is supplied to the head. $P_{B}^{\text {full }}$, $P_{H}^{f u l t}, T_{H}$, and $T_{B}$ are recorded throughout the experiment.

At $t=0$, the power $P_{0}=7.5 \mathrm{~mW}$ is sent during $\Delta t$ $=300 \mathrm{~s}$ in the head by increasing in a single step the power $P_{H}^{f u l l}$. This value corresponds to $1 \mathrm{~kW} / \mathrm{m}^{2}$. It has been chosen because (1) it is the smallest value that provides enough precision in the data; (2) this power is close to the maximum power that can be extracted by the cell base temperature regulation. The initial temperature $T_{0}$ remains the reference for the base temperature regulation PID (i.e., $T_{B}=T_{0}$ within the precision of the PID). During the experiment, the temperature of the base varies only slightly, on the order of 6 $\mathrm{mK}$ (Fig. 2). The integration time of the PID is typically 10 $\mathrm{s}$. This value thus corresponds to the temporal resolution of the transient heat flux measurements.

The heat power $P_{B}$ transferred by the cell is determined as

$$
P_{B}(t)=P_{B}^{f u l l}(t=0)-P_{B}^{f u l l}(t) .
$$

The power $P_{B}(t)$ is thus limited by the value $P_{B}^{f u l l}(t=0)$. 


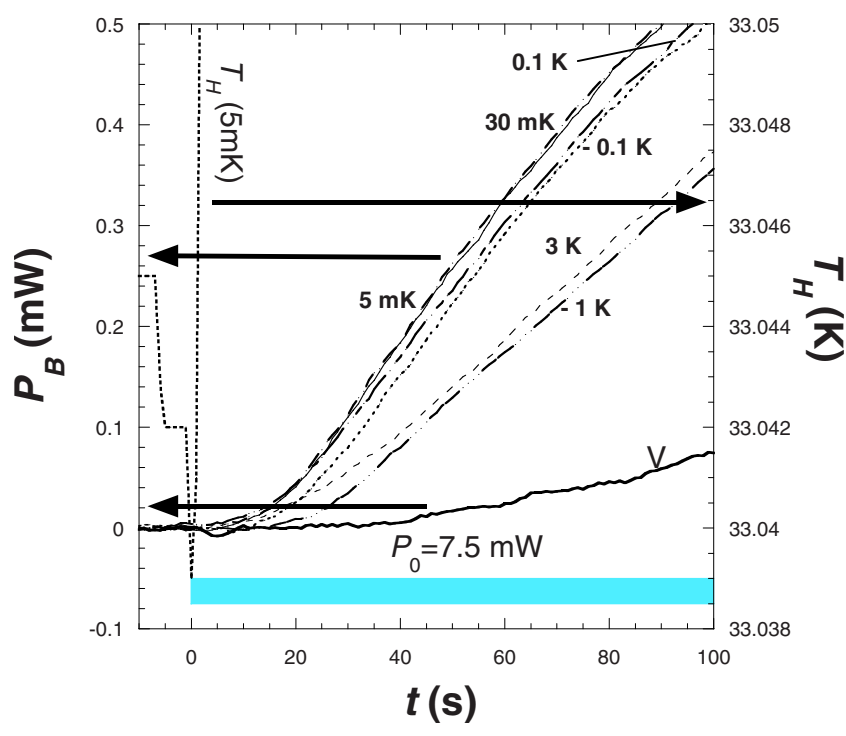

FIG. 3. (Color online) Small time evolution of the transmitted power $\left(P_{B}\right)$ for several temperature distances $T_{0}-T_{c}$, both above and below the critical point. The head temperature evolution (dotted line) is also shown to precisely detect the onset of heating. V: empty cell; $T_{0}-T_{c}=5 \mathrm{mK}$ (solid line), $30 \mathrm{mK}$ (broken line, long dashes), $0.1 \mathrm{~K}$ (double broken line), $3 \mathrm{~K}$ (broken line, short dashes), $-0.1 \mathrm{~K}$ (dotted line), and $-1 \mathrm{~K}$ (dotted-dashed line).

The measurements were initially carried out in the empty cell to evaluate the heat conduction by the PMMA walls. The cell is then filled and measurements are performed at different temperatures $T_{0}$.

\section{Measurement results}

In Fig. 2 typical evolutions of the transmitted power $P_{B}$ and head temperature when the pipe contains $\mathrm{H}_{2}$ initially at $T_{0}=T_{c}+5 \mathrm{mK}$ are shown. The corresponding curves for the empty (under vacuum) cell are shown for comparison. In this latter case, heat is transmitted by the PMMA pipe walls only. One notes that (i) $T_{H}$ is lower when the pipe is filled, which agrees with the higher value of $P_{B}$ and (ii) the dynamics of $P_{B}$ at small time is quite different with empty and filled cells. This is even clearer from Fig. 3 where the typical small time behavior is reported for different temperatures. After the $10 \mathrm{~s}$ reaction time of the thermal regulation, $P_{B}$ increases linearly. In contrast, the heat flux in the empty pipe remains close to zero for long time. The difference in behavior highlights two different mechanisms of heat transfer: (i) thermal diffusion in the walls of the empty pipe where the heat flux is zero before the front of heat diffusion reaches the base and (ii) piston effect in the filled pipe. In the latter case, the dynamics (and the yield, see below) increases when nearing $T_{c}$. The behavior of $P_{B}(t)$ at $T_{0}<T_{c}$ (i.e., when both liquid and gas phases are present in the pipe) is similar to that at $T_{0}>T_{c}$.

The measured time evolution of the transmitted power $P_{B}$ is plotted at different temperatures above and below $T_{c}$ in Fig. 4. One notes that the behavior at $T_{0}<T_{c}$ is similar to the one at $T_{0}>T_{c}$, even very close to $T_{c}$. The yield defined as $Y=P_{B} / P_{0}$ attains at its maximum about $10 \%$. The yield for the filled pipe is always larger than for the empty pipe. At

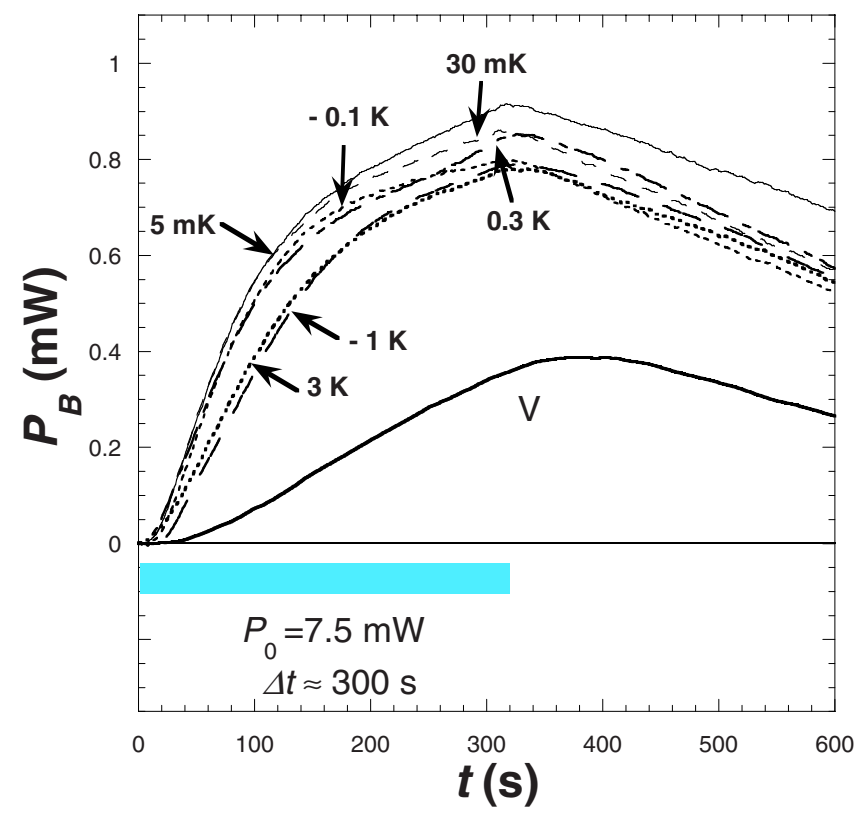

FIG. 4. (Color online) Measured transient of the transmitted power $P_{B}$ for several temperatures above and below the critical point. The time interval during which the power is injected is shown with a bar. V: empty pipe; other curves: filled cell; $T_{0}-T_{c}=5 \mathrm{mK}$ (solid line), $30 \mathrm{mK}$ (broken line, long dashes), $0.1 \mathrm{~K}$ (double broken line), $3 \mathrm{~K}$ (broken line, short dashes), $-0.1 \mathrm{~K}$ (dotted line), and $-1 \mathrm{~K}$ (dotted-dashed line).

large times, the pipe walls contribute to the heat transfer, as seen in Fig. 4, where the rise of $P_{B}$ for the filled cell is roughly parallel to the empty cell curve. The maximum heat flux $\left(P_{B}^{M}\right)$ is then always reached at the end of the heating period.

In order to determine what are the relative contributions of the cell walls and the fluid, it is necessary to analyze the process in great detail. This is performed in Sec. III. It is found that the wall contribution is much smaller than in the empty cell due to the rapid thermalization of the bulk fluid that reduces the temperature gradient in the pipe walls.

It is possible to infer the "initial" slope $d P_{B} / d t$ from Fig. 3. Heat is sent into the cell head at $t=0$ and, after $10-15 \mathrm{~s}, P_{B}$ grows linearly in time during at least 20-30 s. One thus defines as initial slope the value of $d P_{B} / d t$ as measured between 27 and $47 \mathrm{~s}$. During this period, the contribution of the transient conduction by the pipe walls is negligible. The temperature dependence of this slope is reported in Fig. 5(a) for two experimental runs. The data are similar above and below $T_{c}$. The slope increases when going to $T_{c}$ and saturates for $\left|T_{0}-T_{c}\right| \leq 0.3 \mathrm{~K}$. The maximum heat flux (at $300 \mathrm{~s}$ ) $P_{B}^{M}$ (Fig. 4) is plotted with respect to temperature in Fig. 5(b) for two different runs. Data between both runs show a systematic difference of order $15 \%$ that we attribute to the uncertainty of filling of the cell at critical density $( \pm 3 \%)$. They, however, exhibit the same behavior, similar to $d P_{B} / d t$, with an increase when going to $T_{c}$ and saturation for $\left|T_{0}-T_{c}\right|$ $\leq 0.3 \mathrm{~K}$. 

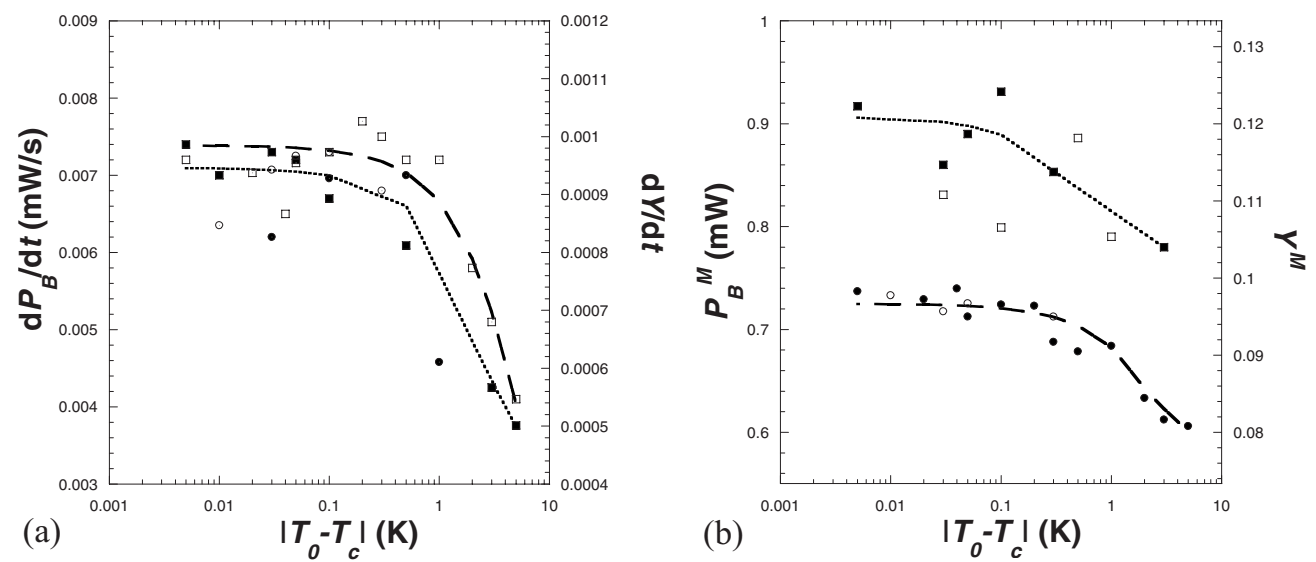

FIG. 5. (a) Measured transient of the transmitted heat flux initial rise $d P_{B} / d t$ (see text) with respect to $\left|T_{0}-T_{c}\right|$. Run 1: circles; run 2: squares. $T_{0}>T_{c}$ : black circles; $T_{0}<T_{c}$ : open circles. The dotted (broken) line is the run 1 (2) smoothing of $T_{0}>T_{c}$ data. (b) Fluid maximum heat transmission $P_{B}^{M}$ (see text) with respect to $\left|T_{0}-T_{c}\right|$. Run 1: circles; run 2: circles. $T_{0}>T_{c}$ : filled circles; $T_{0}<T_{c}$ : open circles. The dotted (broken) line is the run 1 (2) smoothing of $T_{0}>T_{c}$ data.

\section{NUMERICAL SIMULATION IN TWO-DIMENSIONS AND ANALYSIS}

Several important points influence the choice of a numerical method for the simulation.

(i) One-dimensional (1D) numerical simulations are relatively easy to perform. However, in the present study, a 1D simulation would not take into account an important effect, the heat exchange with the lateral walls. Lateral walls are heated by the bulk fluid that is quickly thermalized by the piston effect. This wall influence limits the fluid temperature rise and thus the value of the output heat flux. At least a two-dimensional (2D) numerical simulation is necessary to take this influence into account.

(ii) The near-critical fluid introduces a nonlinearity in our system. Its simulation thus requires an iterative algorithm. On each iteration a complete set of temperature values at each node point should be determined. It means that (i) in practice, the equation of state (EOS), i.e., the relation between fluid temperature $T$, pressure $p$, and density $\rho$, must be solved at each iteration, node point, and time step. It involves an enormous total number of EOS treatments. At the same time, the system is driven quite far from the critical point; the relative temperature distance can be as large as $(T$ $\left.-T_{c}\right) / T_{c} \sim 0.15$. This means that (ii) any simple model EOS (parametric [18], van der Waals, linearized [11], etc.) would not work well. A real EOS for $\mathrm{H}_{2}$ needs to be used in the simulation. The available hydrogen EOS $[12,13]$ is the modified Benedict-Webb-Rubin (MBWR) EOS that includes dozens of terms. The requirements (i) and (ii) make the computation time prohibitively long when the full hydrodynamic description of the supercritical fluid (as in [11]) is used.

Recently, some of us [10] suggested a fast calculation method that results in accurate heat transfer calculations wherever the impact of the fluid advection on the heat transfer can be neglected. It makes use of the boundary element method (BEM) [14] that permits a reduction of the dimensionality of the problem: only the boundary surfaces need to be meshed. Here we use the BEM to simulate this problem in microgravity where the fluid convection can be neglected.

\section{A. Problem statement}

We want to find the amount of heat transferred to the base from the head of the cell while the temperature of the base $T_{B}$ is kept constant. Initially, the temperature of the whole cell is equal to $T_{0}$.

The cell consist of several parts: (i) cell base (denoted by $B$ index), (ii) cell head (denoted by $H$ index), (iii) PMMA tube (denoted by $P A$ index), and (iv) fluid (denoted by $F$ index). These parts need to be modeled separately.

\section{Cell base model}

The cell base is made of electrolytic copper and is assumed isothermal, thanks to its very high thermal conductivity. Its temperature $T_{B}$ is controlled by the temperature regulation and assumed to be constant $\left(=T_{0}\right)$ during each experiment. In reality, the temperature regulation device (see above, Sec. II B) has an integration time $t_{I} \simeq 10 \mathrm{~s}$ that characterizes the time over which it averages the actual base temperature data $T_{B}$ in order to maintain the base temperature at its reference value $T_{0}$. We take into account this regulation "inertia" indirectly by integrating over $t_{I}$ the function $P_{B}(t)$ obtained in the simulation.

The power $P_{B}$ transferred to the base is the main output of the simulation to be compared with the experiment. It is a sum of the heat power that arrives from the cell head by the tube walls and by the fluid column,

$$
P_{B}=-\left.k_{P A} \int_{\left(S_{P A}\right)} \frac{\partial T_{P A}}{\partial \vec{n}}\right|_{B} d S-\left.k_{F} \int_{\left(S_{F}\right)} \frac{\partial T_{F}}{\partial \vec{n}}\right|_{B} d S,
$$

where $k$ denotes the thermal conductivity and the integration is performed over the cross-section areas of the PMMA tube $S_{P A}$ and of the fluid $S_{F}$ (see Fig. 6); the unit normal vector $\vec{n}$ is directed inside the base. Since $T_{B}$ is assumed to be constant in time, the heat power that leaks to the He bath remains constant and $P_{B}$ given by Eq. (2) corresponds to the measured quantity (1). 


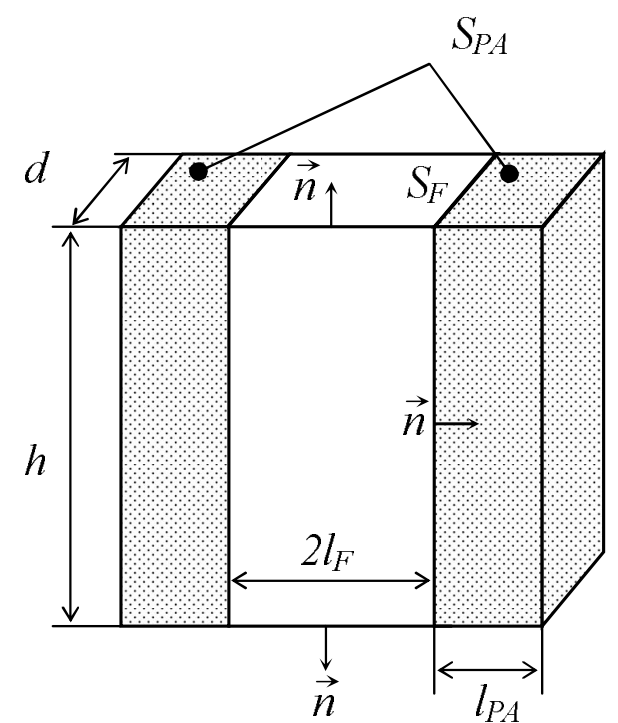

FIG. 6. Scheme of the cell representation in two dimensions (fluid layer sandwiched between the PMMA walls).

\section{Cell head model}

The main part of the head is made of electrolytic copper that can be assumed isothermal, thanks to its very high thermal conductivity at this temperature. The other part is made of stainless steel, which conducts heat much worse than copper (ratio of thermal conductivities of steel and copper is 0.003; see Sec. II A). However, because of its very small thickness, slow temperature evolution, and a good thermal contact with copper, it can also be assumed isothermal.

The power balance of the head can then be written in the form

$$
\begin{aligned}
P_{H}^{f u l l}= & \left.k_{P A} \int_{\left(S_{P A}\right)} \frac{\partial T_{P A}}{\partial \vec{n}}\right|_{H} d S+\left.k_{F} \int_{\left(S_{F}\right)} \frac{\partial T_{F}}{\partial \vec{n}}\right|_{H} d S+C_{H} \frac{d T_{H}}{d t} \\
& +P_{w}^{f u l l}\left(T_{H}\right),
\end{aligned}
$$

where $P_{H}^{f u l l}$ is the controlled heat power supplied to the head. The first two terms at the right-hand side correspond to the heat power transferred to the tube walls and the fluid, respectively. The unit normal vector $\vec{n}$ is chosen to be directed toward the head. The third term reflects the thermal inertia of the head, the head total heat capacity (measured in $\mathrm{J} / \mathrm{K}$ ) being denoted by $C_{H}$. The power $P_{w}^{\text {full }}=P_{w}^{\text {full }}\left(T_{H}\right)$ is the heat leak due to the copper wire that connects the head to the $\mathrm{He}$ bath at temperature $T_{\mathrm{He}}=2.17 \mathrm{~K}$. Due to the high thermal diffusivity of the copper, the temperature distribution in the wire can be assumed to follow immediately the temperature of the head. This implies that

$$
P_{w}^{f u l l}(T)=\frac{S_{w}}{l_{w}} \int_{T_{\mathrm{He}}}^{T} k_{\mathrm{Cu}}(T) d T,
$$

where $S_{w}=0.05 \mathrm{~mm}^{2}$ and $l_{w}=20 \mathrm{~cm}$ are the section area and the length of the wire. Since the copper thermal conductivity $k_{\mathrm{Cu}}$ does not exhibit a strong temperature dependence between 33 and $43 \mathrm{~K}$ (where the experiments are carried out), $P_{w}\left(T_{H}\right)$ can be approximated by the linearized expression

$$
P_{w}^{\text {full }}\left(T_{H}\right) \simeq P_{w}^{\text {full }}\left(T_{0}\right)+\frac{S_{w}}{l_{w}} k_{\mathrm{Cu}}\left(T_{0}\right)\left(T_{H}-T_{0}\right) .
$$

Note that the constant power $P_{w}^{\text {full }}\left(T_{0}\right)$ needs to be supplied to the head both before (to maintain $T_{H}=T_{0}$ ) and during the experiment (as a part of $P_{H}^{\text {full }}$ ) to compensate the heat leaks. In the following, we will denote $P_{H}(t)=P_{H}^{f u l l}(t)-P_{w}^{f u l l}\left(T_{0}\right)$. It represents the excess of the supplied power with respect to its value at $t=0$. In the above experiment, $P_{H}$ was switched on at $t=0$, kept at a constant level $P_{0}$ during the time $\Delta t$, and then switched off.

\section{PMMA wall model}

To estimate correctly the heat exchange between the fluid and the tube, it is important that the heat exchange area $2 \pi R_{i} h$ be reproduced correctly in the simulated $2 \mathrm{D}$ configuration. The fluid layer is supposed to be sandwiched between two plain walls (see Fig. 6) so that the PMMA-fluid contact area is $2 d h$. This condition defines the depth $d=\pi R_{i}$ of the sandwich. The effective fluid layer half-thickness is then $l_{F}$ $=S_{F} / 2 d=R_{i} / 2$. The effective wall thickness is $l_{P A}=S_{P A} / 2 d$. The heat flux and temperature are continuous at the PMMAfluid contact area, where the normal is directed inside the PMMA,

$$
\begin{aligned}
k_{P A} \frac{\partial T_{P A}}{\partial \vec{n}} & =k_{F} \frac{\partial T_{F}}{\partial \vec{n}}, \\
T_{P A} & =T_{F} .
\end{aligned}
$$

Since the cell is in vacuum and the radiative heat transfer is negligible because of the low temperature, the zero flux boundary condition can be defined at the external surface of the cell PMMA walls. The isothermal conditions $T_{P A}=T_{H}$ and $T_{P A}=T_{0}$ are defined at the areas of contact PMMA head and PMMA base, respectively. Notice that while $T_{0}$ remains constant, $T_{H}$ is a function of time. Its evolution is calculated by using Eqs. (4) and (5).

The heat conduction problem has trivial initial conditions

$$
\begin{gathered}
\frac{\partial \varphi}{\partial t}=D_{P A} \nabla^{2} \varphi, \\
\left.\varphi\right|_{t=0}=0,
\end{gathered}
$$

where $D_{P A}$ is the thermal diffusivity of the PMMA. It has to be solved for the reduced temperature $\varphi=T_{P A}(\vec{x}, t)-T_{0}$ (where $\vec{x}$ is the position vector) to find the variation of the temperature $T_{P A}$ inside the PMMA. The parameters $k_{P A}$ and $D_{P A}$ are assumed to be constant.

\section{Fluid model}

Due to the symmetry, only half of the cell through a cut along the axis needs to be simulated, the zero radial flux being imposed at the cell axis. The approach [10] was used in the simulations. We summarize it below.

The fluid temperature satisfies the equation 


$$
\frac{\partial T_{F}}{\partial t}=D_{F} \nabla^{2} T_{F}+g(t),
$$

with

$$
g(t)=\left(1-\frac{c_{v}}{c_{p}}\right) \frac{d \bar{T}}{d t},
$$

where $\bar{T}=\bar{T}(t)$ is the average fluid temperature (not to be confused with space- and time-dependent local fluid temperature $\left.T_{F}\right), c_{v}\left(c_{p}\right)$ is the specific heat of the fluid at constant volume (pressure), and $D_{F}$ is the fluid thermal diffusivity. The initial condition for $T_{F}$ is

$$
\left.T_{F}\right|_{t=0}=T_{0} .
$$

One can note that the term $g(t)$ is only relevant near the critical point where $c_{p} \gg c_{v}$. Since the cell is closed, the mean fluid density $\rho$ in the cell remains constant. The average temperature $\bar{T}$ is defined using the fluid EOS,

$$
\Lambda(p, \rho, \bar{T})=0,
$$

where $p$ is the fluid pressure. To obtain realistic results in a wide temperature interval (in the experiments, the temperature varied within $20 \%$ from $T_{c}$ ), we have to use a realistic EOS. Only the quite complicated NIST MBWR EOS $[12,13]$ is available for parahydrogen. It is unfortunately precise only relatively far from the critical point, typically for $T_{0}-T_{c}$ $>0.5 \mathrm{~K}$. Discrepancies between simulation and experiments can then be expected close to the critical point because the critical divergence of some parameters will not be properly accounted for in the simulation.

Several methods are available to simulate the thermal behavior of the cell (see $[5,15,16])$. However, with such a complicated EOS, only the approach [10] developed by some of us can be used, the others being prohibitively time consuming. The temporal evolution of the average temperature is given directly by the equation first derived in [3], which replaces the more complicated pressure evolution determination,

$$
\frac{d \bar{T}}{d t}=\frac{1}{\rho v c_{v}} \int_{A} k_{F} \frac{\partial T_{F}}{\partial \vec{n}} d A,
$$

where $v=S_{F} h$ is the total fluid volume. The integration is performed over the whole fluid boundary $A . \vec{n}$ is the outward normal to $A$. Instead of the local values for the fluid parameters $\left(c_{v}, c_{p}, k_{F}, \ldots\right)$ we use everywhere their time-dependent spatially averaged counterparts defined. EOS (11) is used to determine the average density $\rho$ and the average temperature $\bar{T}$. The fluid thermal conductivity $k_{F}$ is determined with a correlation implemented in the NIST REFPROP database [13]. Equation (12) has to be complemented with the initial condition

$$
\left.\bar{T}\right|_{t=0}=T_{0} .
$$

\section{Reduction of the fluid problem}

Two more steps must be performed before the numerical procedure can be applied. First, it is convenient to introduce a new $\psi$ variable instead of $T_{F}$,

$$
\psi(\vec{x}, t)=T_{F}(\vec{x}, t)-T_{0}-\int_{T_{0}}^{\bar{T}}\left(1-\frac{c_{v}}{c_{p}}\right) d \bar{T} .
$$

Equation (8) reduces then to the equation

$$
\frac{\partial \psi}{\partial t}=D_{F} \nabla^{2} \psi
$$

One notes that since all the fluid parameters depend on the time-dependent average temperature $\bar{T}$, the fluid thermal diffusivity can be written as

$$
D_{F}=D_{d} f(\bar{T})
$$

and is time dependent. In this equation, the function $f$ is nondimensional and on the order of unity and $D_{d}$ is a constant. To eliminate this time dependence from $D_{F}$ in Eq. (15), one makes a second step by introducing a new variable $\tau$ defined by the equations

$$
\begin{gathered}
\frac{d \tau}{d t}=f(\bar{T}), \\
\left.\tau\right|_{t=0}=0 .
\end{gathered}
$$

Since $\bar{T}$ is a function of $t$ only, the initial value of the problem is fully defined. The substitution of Eqs. (17) into Eq. (15) results in a linear diffusion problem with a constant diffusion coefficient $D_{d}$,

$$
\begin{gathered}
\frac{\partial \psi}{\partial \tau}=D_{d} \nabla^{2} \psi, \\
\left.\psi\right|_{\tau=0}=0 .
\end{gathered}
$$

\section{B. Numerical implementation}

A linear 2D heat diffusion problem can be solved by BEM. Below we outline this method for problems (18) that is equivalent to the following integral equation:

$$
\begin{aligned}
& D_{d} \int_{0}^{\tau} d \tau^{\prime} \int_{A}\left[G\left(\vec{x}-\vec{x}^{\prime}, \tau-\tau^{\prime}\right) q\left(\vec{x}^{\prime}, \tau^{\prime}\right)\right. \\
& \left.\quad-\psi\left(\vec{x}^{\prime}, \tau^{\prime}\right) \frac{\partial_{x^{\prime}} G\left(\vec{x}-\vec{x}^{\prime}, \tau-\tau^{\prime}\right)}{\partial \vec{n}}\right] d_{x^{\prime}} A \\
& =\frac{1}{2} \psi(\vec{x}, \tau),
\end{aligned}
$$

where $q(\vec{x}, \tau)=\partial \psi(\vec{x}, \tau) / \partial \vec{n}$ and $G$ is the Green's function for the infinite space, 


$$
G(\vec{x}, \tau)=\frac{1}{4 \pi \tau D_{d}} \exp \left(-\frac{|\vec{x}|^{2}}{4 \tau D_{d}}\right) .
$$

Equation (19) is solved by breaking the integration contour $A$ into straight boundary elements (BEs). On each of them $\psi$ and $q$ are assumed to be constant and equal to their values at the node point $\vec{x}_{i}$ in the middle of the $i$ th BE. The time interval $(0, \tau)$ is broken into the intervals $\left(\tau_{f-1}, \tau_{f}\right)$ on which $\psi$ and $q$ are assumed to be constant and equal to $\psi_{f i}$ and $q_{f i}$ at the $i$ th BE. Equation (19) then reduces to the set of linear algebraic equations,

$$
\sum_{f=1}^{F} \sum_{j=1}^{N}\left(q_{f j} G_{i j}^{F f}-\psi_{f j} H_{i j}^{F f}\right)=\psi_{F i} / 2,
$$

where $i=1, \ldots, N . H_{i j}$ and $G_{i j}$ are the BEM coefficients,

$$
\begin{gathered}
G_{i j}^{F f}=D_{d} \int_{\tau_{f-1}}^{\tau_{f}} d t \int_{A_{j}} G\left(\vec{x}_{i}-\vec{x}, \tau_{F}-t\right) d_{x} A, \\
H_{i j}^{F f}=D_{d} \int_{\tau_{f-1}}^{\tau_{f}} d t \int_{A_{j}} \frac{\partial G\left(\vec{x}_{i}-\vec{x}, \tau_{F}-t\right)}{\partial \vec{n}_{x}} d_{x} A .
\end{gathered}
$$

Their calculation is discussed in detail in [14]. The $2 N$ variables $\left\{q_{F i}, \psi_{F i}\right\}(i=1, \ldots, N)$ are related via $N$ more equations that correspond to the boundary conditions given at each of the $N$ BEs. The total number of equations is then $2 N$ and corresponds to the total number of the unknowns. This set of equations should be solved sequentially for each time moment $\tau_{F}$, where $F$ varies from 1 to the maximum required value.

The calculation algorithm needs to be iterative to find $\bar{T}$ :

(1) $\bar{T}\left(t_{F-1}\right)$ is taken as the initial guess for $\bar{T}\left(t_{F}\right)\left(T_{0}\right.$ is used for $F=1$ ).

(2) The fluid parameters are calculated for $\bar{T}\left(t_{F}\right)$ using the EOS; $\tau_{F}$ is calculated with Eqs. (17).

(3) Coupled problems (7) and (18) are solved by BEM; the heat flux distribution over the fluid boundary is found.

(4) It is used to determine the corrected value of $\bar{T}\left(t_{F}\right)$ with Eq. (12).

(5) Steps 2-4 are repeated until the convergence is achieved.

(6) Steps 1-5 are repeated for the next time moment.

Since the summation in Eqs. (21) has to be performed over all $f=1, \ldots, F$, the described BEM formulation has a disadvantage of an increase of the calculation volume with $F$. On the other hand, the algorithm is very stable, which permits to choose a relatively large time step with practically no loss in accuracy.

\section{Adjustment of the cell parameters}

First, one needs to check if the model parameters correspond to the actual experimental values. The test consists in comparing the simulation and the experiments in an empty cell of both the evolution of the heat flux and head temperature. This simulation appears to be very sensitive to the precise value of the heat capacity of the head $C_{H}=m_{H} c_{H}$, where

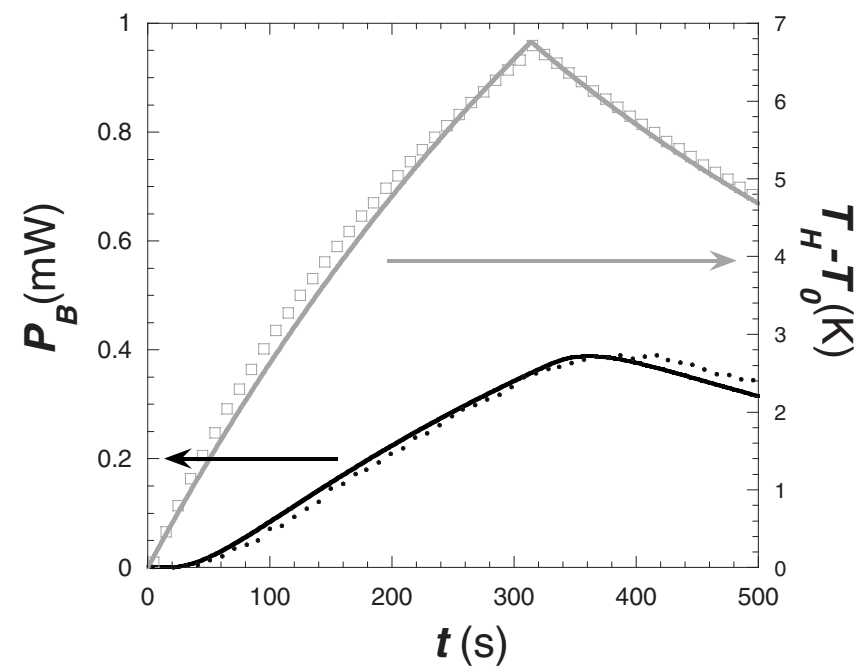

FIG. 7. Empty pipe: comparison between the simulation (lines) and the experiments (characters) for $7.5 \mathrm{~mW}$ injected power. $T_{0}$ $=33 \mathrm{~K}$. Black line and dots: transmitted power $P_{B}$. Gray line and squares: head temperature $T_{H}$.

$m_{H}$ and $c_{H}$ are the mass and the specific heat of the head. The pieces of the head are made of stainless steel and copper, the specific heats of which at $35 \mathrm{~K}$ being close. For simplification, we will take that of copper $\left(c_{H}=42.6 \mathrm{~J} / \mathrm{kg} \mathrm{K}[17]\right)$. A good agreement between the calculation and the experiment (Fig. 7) is found for $m_{H}=6 \mathrm{~g}$. Taking into account that the copper part weight is $2.4 \mathrm{~g}$ and the stainless-steel pieces are about of the same size, this $m_{H}$ value is quite reasonable.

\section{Some analytical results}

\section{Small time asymptotics}

No heat exchange of the fluid with the tube walls is assumed in this section in order to obtain analytical results. At small times, all fluid parameters can be approximated by their values at the initial temperature, i.e., that of the base $T_{0}$. The temperature profile in the fluid is sketched in Fig. 1(c). It shows three regions: hot and cold diffusion boundary layers (HBL and CBL) that form near the extremities of the cell and a nearly isothermal bulk. The thickness of both HBL and CBL can be estimated with the expression $\delta \sim \sqrt{D_{F} t}$, where $D_{F}$ is the fluid thermal diffusivity. This allows the main parameter, i.e., the transferred power to be found in the form

$$
P_{B} \approx S_{F} k_{F}\left(T_{b k}-T_{B}\right) / \delta,
$$

where $T_{b k}$ means the temperature in the bulk of the fluid and $k_{F}$ is the fluid thermal conductivity. $S_{F}\left(=\pi R_{i}^{2}\right)$ is the fluid cross-section area. Unless stated otherwise, $T_{B}=T_{0}$ and is constant. Similar to Eq. (23), the transfer to the fluid power is

$$
P_{H} \approx S_{F} k_{F}\left(T_{H}-T_{b k}\right) / \delta \text {. }
$$

The bulk of the fluid remains isothermal, which means that $\Delta T_{b k}=0$. By making use of Eqs. (8) and (12) one obtains 

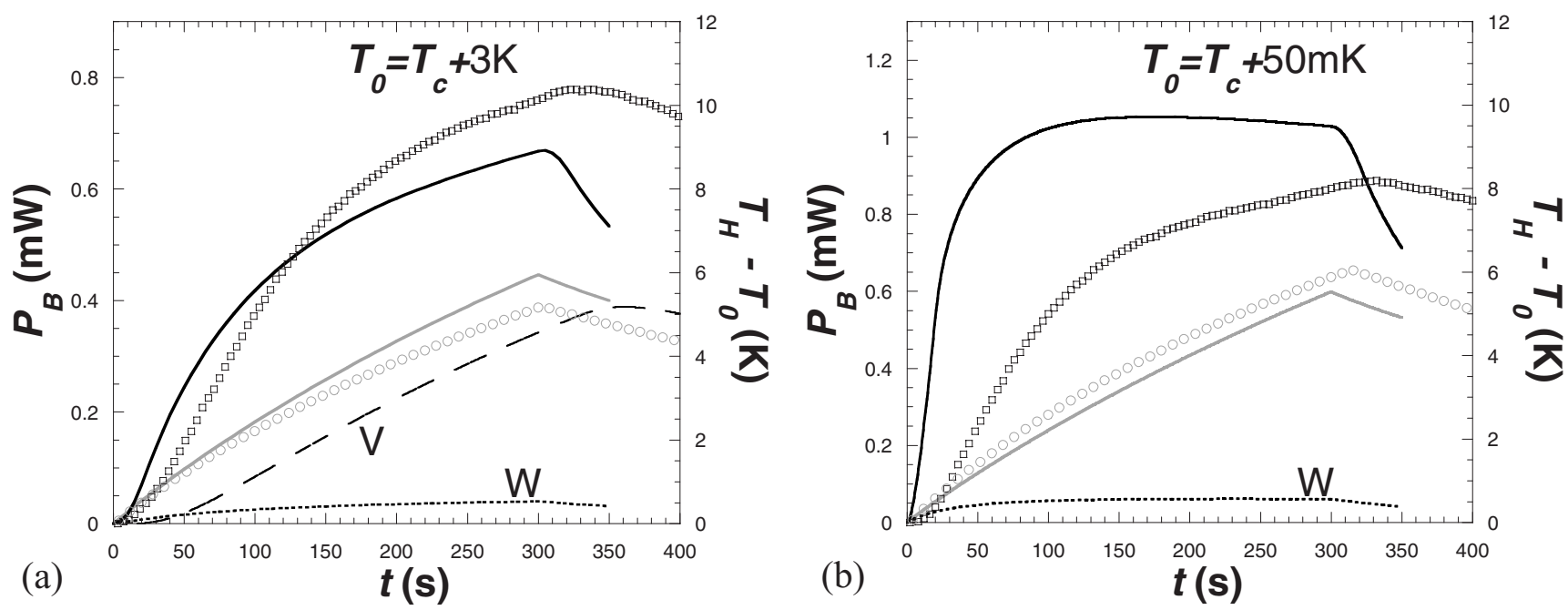

FIG. 8. Comparison between simulation and experiments concerning the evolution of the transmitted heat flux $P_{B}$ (simulation: solid black line; experiment: squares) and head temperature $T_{H}-T_{0}$ (simulation: solid gray line; experiment: circles). Dashed line (V): contribution of the wall conduction for the empty cell (the same as the black curve in Fig. 7), to be compared with the dotted line (W) that represents the contribution of the wall conduction in the filled cell. (a) $T_{0}=T_{c}+3 \mathrm{~K}$. (b) $T_{0}=T_{c}+50 \mathrm{mK}$.

$$
\frac{d T_{b k}}{d t} \approx\left(1-\frac{c_{v}}{c_{p}}\right) \frac{P}{\rho v c_{v}}
$$

where $v=S_{F} h$ is the total fluid volume, $c_{v}\left(c_{p}\right)$ is the specific heat of the fluid at constant volume (pressure), and $P$ is the total heat supplied to the fluid per unit time.

Two kinds of model problems are considered below for the sake of comparison: a constant heat flux boundary condition at the fluid-head interface, the numerical solution for which is analyzed in [10], and a more realistic problem where the heat capacity of the head controls the head temperature evolution. As previously, it is assumed that $T_{B}$ is equal to the initial fluid temperature $T_{0}$.

\section{Constant heat flux boundary condition}

In this case the $P$ value is constant and one obtains from Eqs. (23) and (25) the following small time asymptotics:

$$
\begin{gathered}
T_{b k}-T_{0} \approx\left(\frac{1}{c_{v}}-\frac{1}{c_{p}}\right) \frac{P}{\rho v} t, \\
P_{B} \approx\left(\frac{c_{p}}{c_{v}}-1\right) \frac{P}{h} \sqrt{t D_{F}} .
\end{gathered}
$$

One notices a steep rise of the output power $P_{B}$ with time similarly to [10] with $d P_{B} / d t \rightarrow \infty$ when $t \rightarrow 0$.

\section{Heat capacity-limited regime}

Let us assume that the contributions of the heat fluxes going both into the fluid and into the PMMA walls are negligible in the head heat balance equation [Eq. (3)]. One can then obtain from Eqs. (3) and (5)

$$
P_{H}=C_{H} \frac{d T_{H}}{d t}+\frac{S_{w}}{l_{w}} k_{\mathrm{Cu}}\left(T_{H}-T_{0}\right) .
$$

Since all the parameters in this equation are constant, one can infer from it the time evolution of $T_{H}$,

$$
T_{H}=T_{0}+\frac{P_{H} \tau_{r}}{C_{H}}\left[1-\exp \left(-\frac{t}{\tau_{r}}\right)\right],
$$

where $\tau_{r}=l_{w} C_{H} /\left(S_{w} k_{\mathrm{Cu}}\right) \approx 600 \mathrm{~s}$ is the relaxation time. It appears that this regime applies in our case. Indeed, Fig. 8 below shows a behavior similar to Eq. (29) with a relaxation time on the order of several hundreds of seconds. This implies that if $P_{H}$ was switched on for a long time in our experiment, $T_{H}$ would relax to the value $T_{0}+P_{H} \tau_{r} / C_{H} \approx 50 \mathrm{~K}$ at the time scale of several $\tau_{r}$. Note that this behavior is completely independent of the fluid.

The small time asymptotics $\left(t \ll \tau_{r}\right)$ is linear,

$$
T_{H}-T_{0} \approx \frac{P_{H}}{C_{H}} t .
$$

The total heat supplied to the fluid can be approximated by the following expression [see Eqs. (23) and (24)]:

$$
P=P_{H}-P_{B} \approx S_{F} k_{F}\left(T_{H}-T_{0}\right) / \delta .
$$

Using it and Eq. (25) one obtains the small time asymptotics,

$$
T_{b k}-T_{0} \approx\left(\frac{c_{p}}{c_{v}}-1\right) \frac{P_{H}}{h C_{H}} \sqrt{D_{F}} t^{3 / 2} .
$$

Equation (23) then results in the linear small time behavior for $P_{B}$,

$$
P_{B} \approx\left(\frac{c_{p}}{c_{v}}-1\right) \frac{P_{H} S_{F} k_{F}}{h C_{H}} t .
$$

A comparison of Eq. (27) with Eq. (33) shows that the small time behavior of both $T_{H}$ and $P_{B}$ depends strongly on the boundary condition imposed at the solid-fluid boundary. While the slope $d P_{B} / d t$ is infinite for the constant heat flux injection, it remains finite for the heat injector of finite heat capacity. This conforms to the experimental data. For the sample at critical density, $d P_{B} / d t$ ought to increase as $\left(c_{p} / c_{v}\right) k_{F} \sim\left[\left(T_{0}-T_{c}\right) / T_{c}\right]^{-0.57}$ when approaching $T_{c}$. In the 

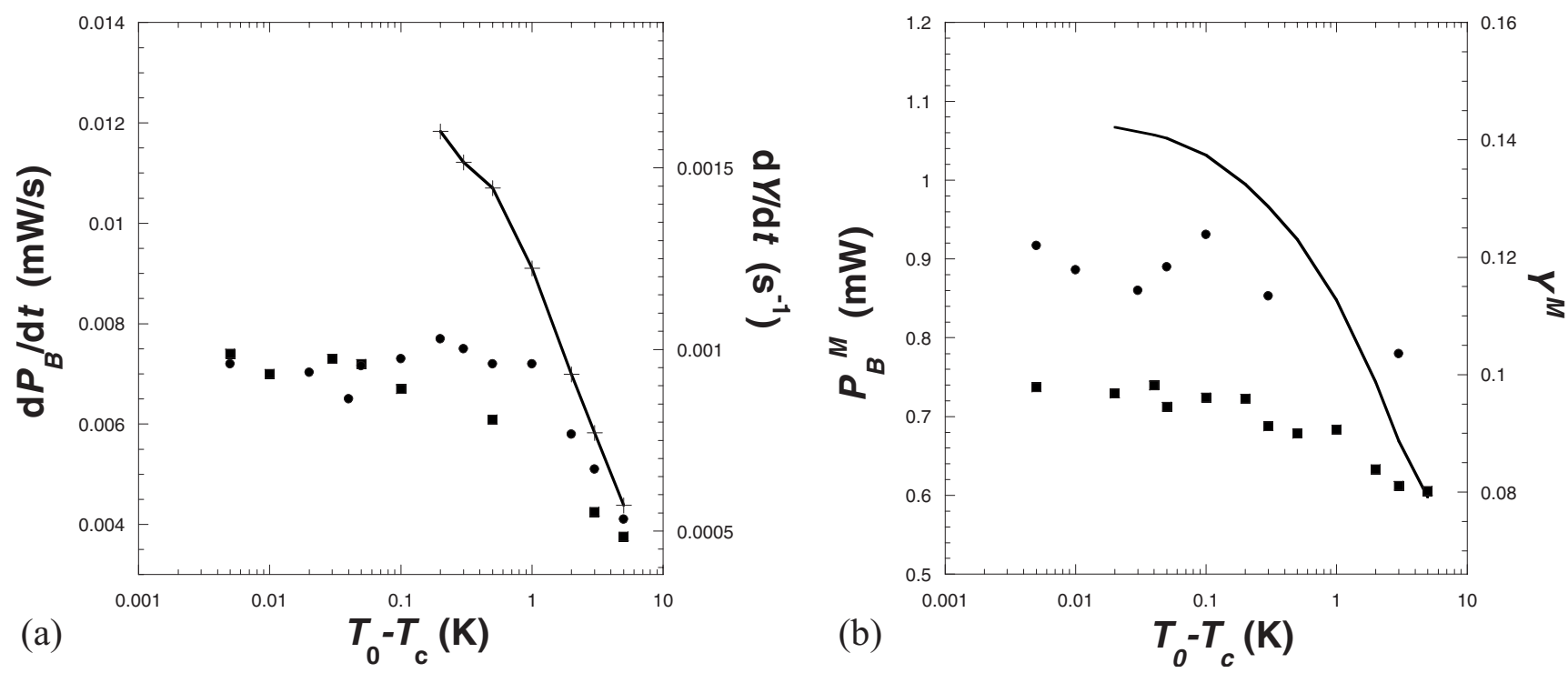

FIG. 9. Comparison (log-log plots) between simulation and experiments concerning the $T-T_{c}$ behavior of (a) the transmitted heat flux initial rise $d P_{B} / d t$ and reduced yield rise $d Y / d t$ (see text). Simulations: solid line and crosses. Experiments: run 1: circles; run 2: squares. (b) maximum heat transmission $P_{B}^{M}$ and $Y^{M}$ (see text). Simulations: line. Experiments: run 1: circles; run 2: squares.

experiments [Fig. 5(a)], a slope increase is indeed observed when approaching $T_{c}$. The increase is followed, however, by a saturation of the slope for $T_{0}-T_{c}<0.3 \mathrm{~K}$. This saturation cannot be attributed to a substantial change of the fluid properties with temperature in the bulk fluid as only the early times are concerned. As a matter of fact, this saturation is not observed in the simulation [see Fig. 9(a) below] where the temperature inhomogeneity is considered rigorously. We thus attribute the saturation to a slightly off-critical density of the cell.

\section{E. Simulation results}

\section{Temperature dependence and scaling}

A simulation is compared to the experiment performed at (i) a large distance from the critical point $T_{0}-T_{c}=3 \mathrm{~K}$ [Fig. 8(a)] and (ii) at a short distance $T_{0}-T_{c}=50 \mathrm{mK}$ [Fig. 8(b)]. One notes that the simulation represents relatively well the temperature evolution of the head with, however, some systematic discrepancies. These differences are also found in the transmitted flux, somewhat smaller in the simulation far from $T_{c}$, corresponding to a larger rise in $T_{H}$, and larger close to $T_{c}$, corresponding to a smaller rise in $T_{H}$. We attribute these discrepancies to the EOS which is not supposed to be correct in the close vicinity of the critical point.

For the sake of comparison, the quantities $P_{B}^{M}$ and $d P_{B} / d t$ for both the experiments and the simulations, obtained as an average slope between 27 and $47 \mathrm{~s}$ (as in Sec. II C), have also been plotted in Fig. 9. $d P_{B} / d t$ evolves strongly in this time range for $T-T_{c}<0.2 \mathrm{~K}$ and for this reason is not plotted. However, it is clear that the initial slope $d P_{B} / d t$ grows as $T-T_{c}$ decreases. The saturation of $P_{B}^{M}$ near $T_{c}$ is probably related to the change of the fluid properties with temperature in the bulk fluid that occur at large times. The agreement is fair if one considers the differences in the two experimental runs and the fact that the EOS apparently overestimates the divergence of the compressibility close to $T_{c}$.

\section{Length dependence}

We consider now the length dependence of the flux yield. The efficiency of the bulk heating produced by the Piston effect is inversely proportional to the fluid volume $v$ [see Eq. (25)]. According to Eq. (33), $P_{B}$ (and thus $Y$ ) should be inversely proportional to $h$ at small times. Although it is obvious that the maximum of yield should decrease with increasing pipe length, it is difficult to obtain the analytical dependence on $h$ at long evolution times and a numerical simulation is necessary. The simulations are carried out for a single temperature $T_{c}+3 \mathrm{~K}$ and different multiples of 16.5 $\mathrm{mm}:(\times 2: 33 \mathrm{~mm} ; \times 5: 82.5 \mathrm{~mm} ; \times 10: 165 \mathrm{~mm})$. The results are reported in Fig. 10. The maximum yield is seen to

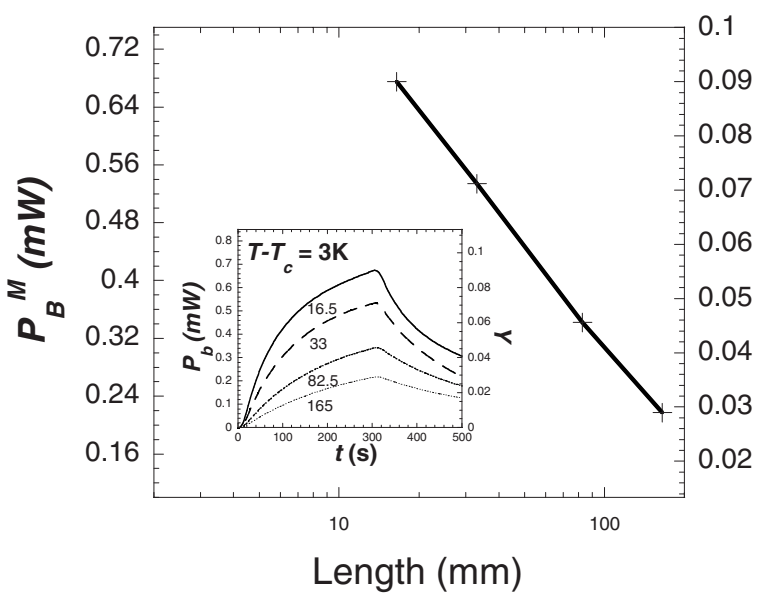

FIG. 10. Variation at $T_{c}+3 \mathrm{~K}$ of the maximum heat flux $P_{B}^{M}$ and corresponding yield $Y^{M}$ with respect to cell length (semilogarithmic scale) for $7.5 \mathrm{~mW}$ injected power. In the insert the evolution for the different lengths is shown. 

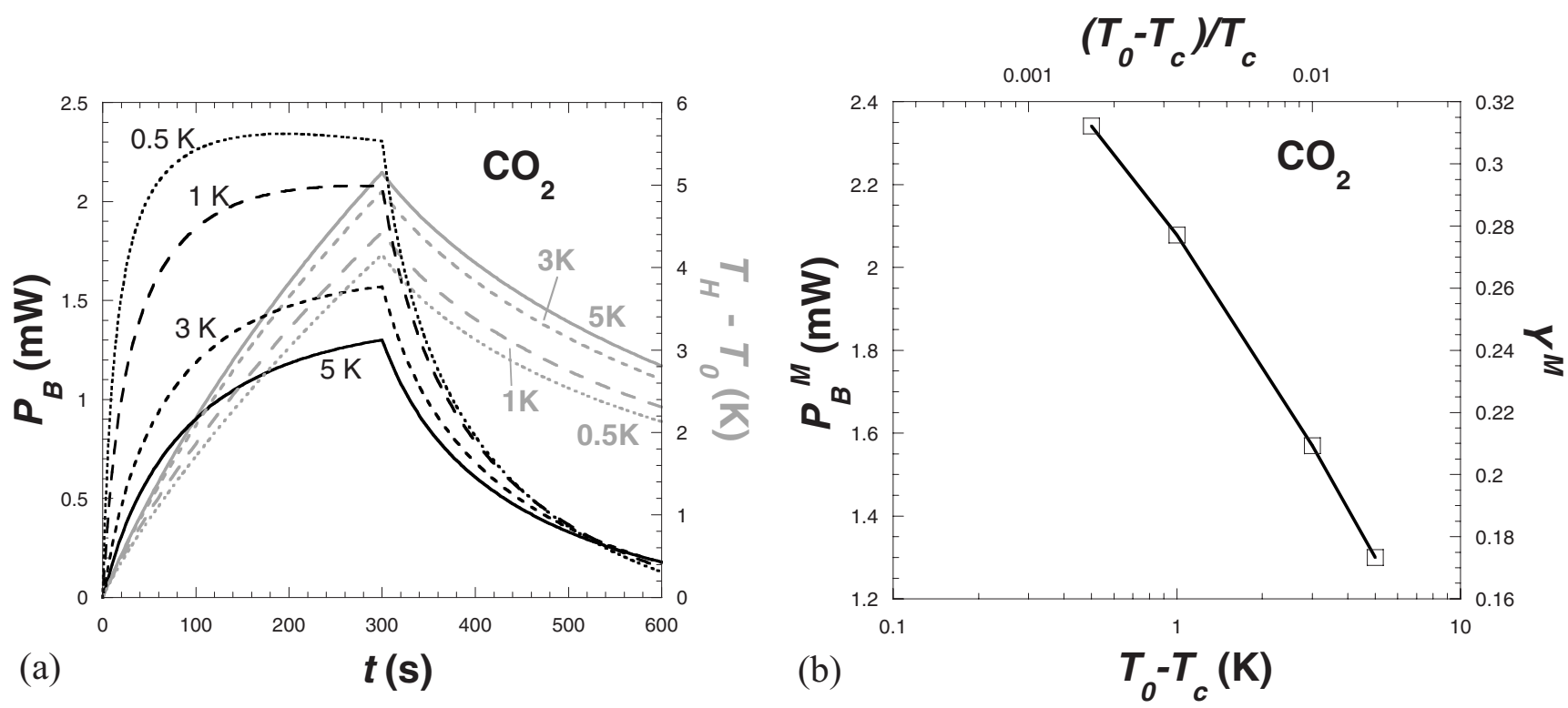

FIG. 11. (a) $\mathrm{CO}_{2}$ heat pipe simulation for $7.5 \mathrm{~mW}$ injected power. (a) Evolution of the transmitted heat flux $P_{B}$ (black curves) and head temperature $T_{H}-T_{0}$ (gray curves) for different base temperatures: $T_{0}-T_{c}=5 \mathrm{~K}$ (solid line), $3 \mathrm{~K}$ (broken line, short dashes), $1 \mathrm{~K}$ (broken line, long dashes), and $0.5 \mathrm{~K}$ (dotted line). (b): Semilogarithmic plot of the maximum heat flux $P_{B}^{M}$ and corresponding yield $Y^{M}$ with respect to $T_{0}-T_{c}$ (lower abscissa) or to the reduced temperature $\left(T_{0}-T_{c}\right) / T_{c}$ (upper abscissa).

decrease according to a law close to logarithmic $Y^{M}$ $=Y_{0} \ln \left(h_{M} / h\right)$, with $Y_{0}=0.0267 \pm 0.0006$ and $h_{M}=475 \pm 23$. The reason for such a law is unclear.

\section{Generalization to $\mathrm{CO}_{2}$}

The simulation realized for the same parameters can be applied to another fluid, $\mathrm{CO}_{2}$, whose critical temperature is $T_{c}=304 \mathrm{~K}$. The restricted cubic parametric EOS [18] has been used to describe $\mathrm{CO}_{2}$. For the sake of comparison, the material parameters of the cell solid cell components were chosen to be the same as for the experimental cell at $30 \mathrm{~K}$. The simulation is carried out for four temperature values $\left(T_{0}=T_{c}+5,3,1,0.5 \mathrm{~K}\right)$, which correspond to about the same reduced temperatures $\left(T_{0}-T_{c}\right) / T_{c}$ as $\mathrm{H}_{2}$ at $T_{c}+0.5 \mathrm{~K}$, $0.3 \mathrm{~K}, 100 \mathrm{mK}$, and $50 \mathrm{mK}$. The results are shown in Fig. 11. The yield is larger with respect to that of $\mathrm{H}_{2}$. In particular, it is of order $30 \%$ at $T_{0}-T_{c}=1 \mathrm{~K}$, whereas it is about $10 \%$ for $\mathrm{H}_{2}$ at $T_{0}-T_{c}=0.11 \mathrm{~K}$, which corresponds to the same reduced temperature $(0.033)$. This can be explained by the fact that the same power causes a smaller rise in reduced temperature so that the ratio of (fluid heat capacity) and (head heat capacity) is larger for $\mathrm{CO}_{2}$ than for $\mathrm{H}_{2}$.

\section{CONCLUSION}

This study aimed to determine whether the piston effect in near-critical fluids could be used to transport heat at large distance, as heat pipes do. The yield defined as the ratio of the injected and transported heat power does not exceed 10-30\%. It also decreases with the tube length (nevertheless, with a slow logarithmic decay). An interesting transport property, however, has been found. Any change in injected power is transmitted very rapidly, with a rate that is only limited by the heat capacity of the cell. Although the experiments (and most simulations) were performed with $\mathrm{H}_{2}$, the conclusions are applicable to any other fluid. In particular, they are valid for $\mathrm{CO}_{2}$, which was studied numerically. This fast heat transmission exists in a large temperature range around the critical point $\left(10 \mathrm{~K}\right.$ in $\mathrm{H}_{2}$ and $100 \mathrm{~K}$ in $\left.\mathrm{CO}_{2}\right)$. This means that a tube filled with such (appropriately chosen according to the temperature range) fluid can be used as a thermal link installed in parallel with a conventional heat pipe. Such a tube filled with fluid is an efficient thermal link during fast changes of the applied power.

\section{ACKNOWLEDGMENTS}

The authors are grateful to Eric W. Lemmon from NIST for giving us his numerical code for the parahydrogen MBWR EOS and the transport coefficients. We thank Hélène Félice for her help during the experimental part of this work. A partial financial support by CNES is acknowledged. 
[1] B. Zappoli, D. Bailly, Y. Garrabos, B. Le Neindre, P. Guenoun, and D. Beysens, Phys. Rev. A 41, 2264 (1990).

[2] A. Onuki, H. Hao, and R. A. Ferrell, Phys. Rev. A 41, 2256 (1990).

[3] A. Onuki and R. A. Ferrell, Physica A 164, 245 (1990).

[4] P. Guenoun, B. Khalil, D. Beysens, Y. Garrabos, F. Kammoun, B. Le Neindre, and B. Zappoli, Phys. Rev. E 47, 1531 (1993).

[5] A. B. Kogan and H. Meyer, Phys. Rev. E 63, 056310 (2001).

[6] R. de Bruijn, R. J. J. van Diest, T. D. Karapantsios, A. C. Michels, W. A. Wakeham, and J. P. M. Trusler, Physica A 242, 119 (1997).

[7] V. S. Nikolayev, D. Chatain, D. Beysens, and G. Pichavant, Microgravity Sci. Technol., doi:10.1007/s12217-010-9217-6 (2010).

[8] R. Wunenburger, D. Chatain, Y. Garrabos, and D. Beysens, Phys. Rev. E 62, 469 (2000).

[9] D. Chatain and V. S. Nikolayev, Cryogenics 42, 253 (2002).

[10] V. S. Nikolayev, A. Dejoan, Y. Garrabos, and D. Beysens, Phys. Rev. E 67, 061202 (2003).
[11] B. Zappoli, A. Jounet, S. Amiroudine, and A. Mojtabi, J. Fluid Mech. 388, 389 (1999).

[12] B. A. Younglove, J. Phys. Chem. Ref. Data Suppl. 1, 1 (1982); 14, 619(E) (1985).

[13] NIST chemistry webbook, NIST standard reference database 12, (http://webbook.nist.gov).

[14] V. S. Nikolayev and D. A. Beysens, in Boundary Elements XXIV (Incorporating Meshless Solutions), International Series on Advances in Boundary Elements Vol. 13, edited by C. A. Brebbia, A. Tadeu, and V. Popov (WIT Press, Southampton, 2002) pp. 501-525.

[15] B. Zappoli, S. Amiroudine, P. Carles, and J. Ouazzani, J. Fluid Mech. 316, 53 (1996).

[16] H. Boukari, J. N. Shaumeyer, M. E. Briggs, and R. W. Gammon, Phys. Rev. A 41, 2260 (1990).

[17] D. L. Martin, Rev. Sci. Instrum. 58, 639 (1987).

[18] M. R. Moldover, J. V. Sengers, R. W. Gammon, and R. J. Hocken, Rev. Mod. Phys. 51, 79 (1979). 